\title{
TAYLOR OBSTRUCTION TO THE INTEGRABILITY OF HOMOGENEOUS POTENTIALS OF DEGREE TWO. AN APPLICATION OF HIGHER ORDER VARIATIONAL EQUATIONS
}

\author{
GUILLAUME DUVAL \\ Laboratoire de Mathématiques et d'Informatique (LMI), INSA de Rouen \\ Avenue de l'Université 76 801, Saint Etienne du Rouvray Cedex, France \\ E-mail: guillaume.duval@insa-rouen.fr \\ ANDRZEJ J. MACIEJEWSKI \\ J. Kepler Institute of Astronomy, University of Zielona Góra \\ Licealna 9, 65-417, Zielona Góra, Poland \\ E-mail: maciejka@astro.ia.uz.zgora.pl
}

We dedicate this paper to Michael Singer on his $60^{\text {th }}$ birthday for his pioneering works in Galois obstruction to integrability. Moreover, we want to thank him for his constant and inspiring influence on our way of thinking.

Abstract. We show how using the differential Galois theory one can find effectively necessary conditions for the integrability of Hamiltonian systems with homogeneous potentials.

1. Introduction. We consider complex Hamiltonian system defined in a simply connected open set in $\mathbb{C}^{2 n}$ by a holomorphic Hamiltonian function $H=H(\boldsymbol{q}, \boldsymbol{p})$, where $\boldsymbol{q}=\left(q_{1}, \ldots, q_{n}\right)$ and $\boldsymbol{p}=\left(p_{1}, \ldots, p_{n}\right)$ denote the canonical coordinates and the momenta, respectively. For such systems the equations of motion have the canonical form

$$
\frac{\mathrm{d}}{\mathrm{d} t} \boldsymbol{q}=\frac{\partial H}{\partial \boldsymbol{p}}(\boldsymbol{q}, \boldsymbol{p}), \quad \frac{\mathrm{d}}{\mathrm{d} t} \boldsymbol{p}=-\frac{\partial H}{\partial \boldsymbol{q}}(\boldsymbol{q}, \boldsymbol{p}),
$$

where $t$ denotes the complex time. In order to simplify notation we denote $\boldsymbol{z}=(\boldsymbol{q}, \boldsymbol{p})$, and rewrite the above system in the form

$$
\frac{\mathrm{d}}{\mathrm{d} t} \boldsymbol{z}=\boldsymbol{X}_{H}(\boldsymbol{z})
$$

2010 Mathematics Subject Classification: 70Hxx, 37J30.

Key words and phrases: differential Galois theory, integrability, non-integrability, Hamilton systems.

The paper is in final form and no version of it will be published elsewhere. 
Let $\varphi(t)$ be a particular solution of the above equation and $\boldsymbol{\Gamma}$ the corresponding phase curve. The variational equation along $\boldsymbol{\Gamma}$ has the form

$$
\frac{\mathrm{d}}{\mathrm{d} t} \boldsymbol{x}=\boldsymbol{A} \boldsymbol{x}, \quad \text { where } \quad \boldsymbol{A}=\frac{\partial \boldsymbol{X}_{H}}{\partial \boldsymbol{z}}(\boldsymbol{\varphi}(t)) .
$$

To this equation two groups are attached: the monodromy group $M$ and the differential Galois group $G$. Because of the Hamiltonian nature of the equation both these groups are subgroups of $\operatorname{Sp}(2 n, \mathbb{C})$. Properties of these groups reflect properties of solutions of the variational equation, and, to some extent, properties of nonlinear equation (2). For example, as is well known, if equation (2) admits a holomorphic (meromorphic) first integral $F(\boldsymbol{z})$, then the variational equation has a polynomial (rational) first integral $f(\boldsymbol{z})$. This implication is basic for an application of the differential Galois theory to finding necessary conditions of integrability. Simply, the existence of a first integral of linear equations implies that its differential Galois group admits a rational invariant. Thus, for integrable system the differential Galois group of the variational equation cannot be too big.

In particular, if the system considered is integrable in the Liouville sense with meromorphic first integrals, then the identity component of the differential Galois group of the variational equation along a particular solution is Abelian, see, for example, [5, 6]. As numerous applications show, this gives a very strong necessary condition for integrability. For an overview of these results, see [7].

In spite of great successes of this differential Galois approach one can ask for stronger tools. In fact, there exist examples of evidently non-integrable systems for which the above mentioned necessary conditions for the integrability are fulfilled. Moreover, a true need for a stronger condition for integrability appears in investigations of systems depending on parameters.

In [8], see also [2], it was proposed to apply the described ideas for variational equations of arbitrary order. The simplest way to derive higher order variational equations is the following. We represent a solution of equation (2) as a formal series

$$
\boldsymbol{z}=\boldsymbol{\varphi}(t)+\varepsilon \boldsymbol{z}_{1}+\frac{1}{2 !} \varepsilon^{2} \boldsymbol{z}_{2}+\cdots,
$$

where $\varepsilon$ is a formal parameter. Inserting this expansion into equation (2) and comparing coefficients of the same powers of $\varepsilon$, we obtain an infinite chain of equations

$$
\frac{\mathrm{d}}{\mathrm{d} t} \boldsymbol{z}_{p}=\boldsymbol{A} \boldsymbol{z}_{p}+\boldsymbol{f}_{p}\left(\boldsymbol{z}_{1}, \ldots, \boldsymbol{z}_{p-1}\right), \quad p=1,2, \ldots,
$$

where $\boldsymbol{f}_{1}=\mathbf{0}$. Each of equations in this chain has the same homogeneous part which coincides with the right hand sides of variational equation (3). Term $\boldsymbol{f}_{p}$ in the right hand side of $p$-th variational equation (denoted $\mathrm{VE}_{p}$ ) depends on solutions of previous equations in the chain. As it was shown in [8], there is an appropriate framework allowing to define the differential Galois group of $\mathrm{VE}_{p}$ with $p \in \mathbb{N}$. Moreover, the following theorem was proved.

Theorem 1.1 (Morales-Ramis-Simó, [8]). Assume that a holomorphic Hamiltonian system is integrable in the Liouville sense with the first integrals which are meromorphic in a connected neighbourhood $U$ of the phase curve $\boldsymbol{\Gamma}$ corresponding to a non-equilibrium 
solution, and are functionally independent in $U \backslash \boldsymbol{\Gamma}$. Then, for each $p \in \mathbb{N}$, the identity component $\left(G_{p}\right)^{\circ}$ of the differential Galois group $G_{p}$ of $p$-th order variational equations $\mathrm{VE}_{p}$ along $\boldsymbol{\Gamma}$ is Abelian.

Another proof of this theorem can be found in [2].

There are only a few examples of application of the above theorem with $p>1$, see [7. The reason is that the $\mathrm{VE}_{p}$ with $p \geq 2$ are much more complicated systems than $\mathrm{VE}_{1}$. This is why no systematic studies of higher variational equations have been made.

In 4 we have initiated a general program of a systematic study of general structure of higher order variational equations and their Galois groups in a context of natural Hamiltonian systems with homogeneous potentials. The motivation for this choice was the following. In order to apply the above Theorem 1.1 one has to know a particular phase curve of the relevant system. Generally it is not easy to find a particular solution of a given system of nonlinear differential equations. However, for natural Hamiltonian systems with homogeneous potentials we can find particular solutions in a systematic way. In fact, let us consider a system with Hamiltonian function of the following form

$$
H=\frac{1}{2} \sum_{i=1}^{n} p_{i}^{2}+V(\boldsymbol{q}), \quad \boldsymbol{q}=\left(q_{1}, \ldots, q_{n}\right),
$$

where $V$ is a homogeneous function of degree $k \in \mathbb{Z}^{\star}:=\mathbb{Z} \backslash\{0\}$. For it the equations of motion have the canonical form

$$
\frac{\mathrm{d}}{\mathrm{d} t} \boldsymbol{q}=\boldsymbol{p}, \quad \frac{\mathrm{d}}{\mathrm{d} t} \boldsymbol{p}=-V^{\prime}(\boldsymbol{q}),
$$

where $V^{\prime}(\boldsymbol{q}):=\operatorname{grad} V(\boldsymbol{q})$. Now, let a non-zero vector $\boldsymbol{d} \in \mathbb{C}^{n}$ be a solution of the equation

$$
V^{\prime}(\boldsymbol{d})=\gamma \boldsymbol{d}, \quad \text { where } \quad \gamma \in \mathbb{C}^{\star} .
$$

Such a vector is called a proper Darboux point of the potential $V$. It defines a two dimensional plane in the phase space $\mathbb{C}^{2 n}$, given by

$$
\Pi(\boldsymbol{d}):=\left\{(\boldsymbol{q}, \boldsymbol{p}) \in \mathbb{C}^{2 n} \mid \boldsymbol{q}=\varphi \boldsymbol{d}, \boldsymbol{p}=\psi \boldsymbol{d}, \quad(\varphi, \psi) \in \mathbb{C}^{2}\right\} .
$$

This plane is invariant with respect to the system (7). Equations (7) restricted to $\Pi(\boldsymbol{d})$ have the form of one degree of freedom Hamilton's equations

$$
\frac{\mathrm{d}}{\mathrm{d} t} \varphi=\psi, \quad \frac{\mathrm{d}}{\mathrm{d} t} \psi=-\gamma \varphi^{k-1},
$$

with the following phase curves:

$$
\Gamma_{k, e}:=\left\{(\varphi, \psi) \in \mathbb{C}^{2} \mid \frac{1}{2} \psi^{2}+\frac{\gamma}{k} \varphi^{k}=e\right\} \subset \mathbb{C}^{2}, \quad e \in \mathbb{C} .
$$

In this way, a solution $(\varphi, \psi)=(\varphi(t), \psi(t))$ of 10$)$ gives rise to a solution $(\boldsymbol{q}(t), \boldsymbol{p}(t)):=$ $(\varphi \boldsymbol{d}, \psi \boldsymbol{d})$ of equations 77$)$ with the corresponding phase curve

$$
\boldsymbol{\Gamma}_{k, e}:=\left\{(\boldsymbol{q}, \boldsymbol{p}) \in \mathbb{C}^{2 n} \mid(\boldsymbol{q}, \boldsymbol{p})=(\varphi \boldsymbol{d}, \psi \boldsymbol{d}),(\varphi, \psi) \in \Gamma_{k, e}\right\} \subset \Pi(\boldsymbol{d}) .
$$

In this context significant obstructions to integrability were obtained just by dealing with the first variational equations $\mathrm{VE}_{1}$. However, as we proved in [3], for $k= \pm 2$ no obstruction can be found on the level of $\mathrm{VE}_{1}$, since the differential Galois group of this equation $\mathrm{Gal}\left(\mathrm{VE}_{1}\right)$ is virtually Abelian. 
Here we report one of our results obtained for homogeneous potentials of degree $k=2$.

TheOREM 1.2. Let $V$ be a homogeneous potential of degree $k=2$, satisfying the following assumptions:

1. it has a proper Darboux point, i.e., there exists a non-zero vector $\boldsymbol{d}$ such that $V^{\prime}(\boldsymbol{d})=\gamma \boldsymbol{d}$ with $\gamma \in \mathbb{C}^{\star}$

2. the Hessian matrix $V^{\prime \prime}(\boldsymbol{d})$ is diagonalisable with eigenvalues

$$
\lambda_{1}=\omega_{1}^{2}, \ldots, \lambda_{n}=\omega_{n}^{2},
$$

such that $\omega_{1}, \ldots, \omega_{n}$ are $\mathbb{Z}$-linearly independent; that is, the equality

$$
\boldsymbol{\alpha} \cdot \boldsymbol{\omega}:=\sum_{i=1}^{n} \alpha_{i} \omega_{i}=0, \quad \text { with } \quad \boldsymbol{\alpha} \in \mathbb{Z}^{n},
$$

implies that $\boldsymbol{\alpha}=\mathbf{0}$.

3. The Hamiltonian system (7) with potential $V$ is integrable in the Liouville sense. Then

$$
V(\boldsymbol{q})=\frac{1}{2} \boldsymbol{q}^{T} V^{\prime \prime}(\boldsymbol{d}) \boldsymbol{q} .
$$

In other words, in an appropriate eigenbasis of $V^{\prime \prime}(\boldsymbol{d})$, the Hamiltonian has the form

$$
H=\frac{1}{2} \sum_{i=1}^{n}\left(p_{i}^{2}+\omega_{i}^{2} q_{i}^{2}\right) .
$$

The interpretation of this theorem is the following. The particular solution considered is periodic. In a neighbourhood of this solution we can perform the Birkhoff normalisation, see, e.g., the first two chapters in [1]. It appears that if the system is integrable and the non-resonance condition is fulfilled then the system is linear, and equivalent to the classical oscillator.

Notice that on the one hand the assumptions of the theorem are minimal in the sense that they put restrictions on the linear part of the vector field. On the other hand the non-resonance condition 13 is very strong.

We started our analysis of higher order variational equations for systems with homogeneous potential just from the case of potential of degree two because, as we mentioned already, the first variational equations do not give any restriction. Moreover, in this case the particular solution as well as solutions of the first variational equations are given by trigonometric functions. These facts simplify considerably the analysis.

In the rest of this paper we present the basic ingredients of the proof which is present with all details in 4 .

2. Structure of higher order variational equation along a Darboux point. Here we assume that the Hamiltonian system (7) has a particular solution $(\boldsymbol{q}(t), \boldsymbol{p}(t)):=$ $(\varphi \boldsymbol{d}, \dot{\varphi} \boldsymbol{d})$ associated to a proper Darboux point $\boldsymbol{d}$ of the potential $V$ which is homogeneous of degree $k$. We can rewrite equations (7) in the form of Newton equations

$$
\ddot{\boldsymbol{q}}=\boldsymbol{F}(\boldsymbol{q}),
$$


where $\boldsymbol{F}(\boldsymbol{q})=-V^{\prime}(\boldsymbol{q})$. We put

$$
\boldsymbol{q}=\boldsymbol{q}_{0}+\varepsilon \boldsymbol{q}_{1}+\frac{1}{2 !} \varepsilon^{2} \boldsymbol{q}_{2}+\frac{1}{3 !} \varepsilon^{3} \boldsymbol{q}_{3}+\cdots,
$$

where $\boldsymbol{q}_{0}=\varphi(t) \boldsymbol{d}$, and $\varepsilon$ is a formal small parameter. Inserting the above expansion into equation 16 and comparing terms of the same order with respect to $\varepsilon$ we obtain an infinite sequence of equations. The first of them, $\ddot{\boldsymbol{q}}_{0}=\boldsymbol{F}\left(\boldsymbol{q}_{0}\right)$, is identically satisfied by assumptions. For further purposes we need the next three equations:

$$
\begin{aligned}
& \ddot{\boldsymbol{q}}_{1}=\boldsymbol{F}^{\prime}\left(\boldsymbol{q}_{0}\right) \boldsymbol{q}_{1}, \\
& \ddot{\boldsymbol{q}}_{2}=\boldsymbol{F}^{\prime}\left(\boldsymbol{q}_{0}\right) \boldsymbol{q}_{2}+\boldsymbol{F}^{\prime \prime}\left(\boldsymbol{q}_{0}\right)\left(\boldsymbol{q}_{1}, \boldsymbol{q}_{1}\right), \\
& \ddot{\boldsymbol{q}}_{3}=\boldsymbol{F}^{\prime}\left(\boldsymbol{q}_{0}\right) \boldsymbol{q}_{3}+3 \boldsymbol{F}^{\prime \prime}\left(\boldsymbol{q}_{0}\right)\left(\boldsymbol{q}_{1}, \boldsymbol{q}_{2}\right)+\boldsymbol{F}^{(3)}\left(\boldsymbol{q}_{0}\right)\left(\boldsymbol{q}_{1}, \boldsymbol{q}_{1}, \boldsymbol{q}_{1}\right) .
\end{aligned}
$$

From the above system the following structure of $\mathrm{VE}_{p}$ follows. $\mathrm{VE}_{1}$ is a linear homogeneous equation given by (18). In contrast, the $\mathrm{VE}_{p}$ with $p \geq 2$ are generally nonhomogeneous linear systems. Their linear parts are the same as that of $\mathrm{VE}_{1}$. Moreover, the second term in $\mathrm{VE}_{2}$ is a quadratic form in the solutions of $\mathrm{VE}_{1}$. A bigger complexity appears in $\mathrm{VE}_{3}$ given by 20 . The second term in the right hand side of this equation is a bilinear form in solutions of $\mathrm{VE}_{1}$ and $\mathrm{VE}_{2}$, while the third term is a cubic form in solutions of $\mathrm{VE}_{1}$. As a consequence, for the Picard-Vessiot extension $\mathrm{PV}_{p}$ of $\mathrm{VE}_{p}$ we get the tower of inclusions

$$
K \subset \mathrm{PV}_{1} \subset \mathrm{PV}_{2} \subset \cdots \subset \mathrm{PV}_{p} \subset \mathrm{PV}_{p+1} \subset \cdots
$$

Let us assume that the Hessian matrix $V^{\prime \prime}(\boldsymbol{d})$ is diagonalisable. Then, without loss of generality, we can assume that it is diagonal, and we put $V^{\prime \prime}(\boldsymbol{d})=\operatorname{diag}\left(\lambda_{1}, \ldots, \lambda_{n}\right)$. Let us denote also

$$
\boldsymbol{q}_{j}=\left(q_{1, j}, \ldots, q_{n, j}\right), \quad \text { for } \quad j \in \mathbb{N} .
$$

Then, system of equations (18), 19) and 20ads

$$
\begin{aligned}
& \ddot{q}_{i, 1}=-\lambda_{i} \varphi(t)^{k-2} q_{i, 1}, \\
& \ddot{q}_{i, 2}=-\lambda_{i} \varphi(t)^{k-2} q_{i, 2}+\varphi(t)^{k-3} \Theta^{i}\left(\boldsymbol{q}_{1}, \boldsymbol{q}_{1}\right), \\
& \ddot{q}_{i, 3}=-\lambda_{i} \varphi(t)^{k-2} q_{i, 3}+3 \varphi(t)^{k-3} \Theta^{i}\left(\boldsymbol{q}_{1}, \boldsymbol{q}_{2}\right)+\varphi(t)^{k-4} \Xi^{i}\left(\boldsymbol{q}_{1}\right)
\end{aligned}
$$

where $1 \leq i \leq n$, and $\Theta^{i}$, and $\Xi^{i}$ are polynomials of their arguments

$$
\Theta^{i}\left(\boldsymbol{q}_{1}, \boldsymbol{q}_{2}\right)=\sum_{\alpha, \beta=1}^{n} \theta_{\alpha, \beta}^{i} q_{\alpha, 1} q_{\beta, 2},
$$

where

$$
\theta_{\alpha, \beta}^{i}=\partial_{\alpha} \partial_{\beta} F_{i}(\boldsymbol{d})=-\partial_{\alpha} \partial_{\beta} \partial_{i} V(\boldsymbol{d})=-\frac{\partial^{3} V(\boldsymbol{d})}{\partial q_{\alpha} \partial q_{\beta} \partial q_{i}}
$$

and

$$
\Xi^{i}\left(\boldsymbol{q}_{1}\right):=\sum_{\alpha, \beta, \gamma=1}^{n} \xi_{\alpha, \beta, \gamma}^{i} q_{\alpha, 1} q_{\beta, 1} q_{\gamma, 1}
$$

where

$$
\xi_{\alpha, \beta, \gamma}^{i}=\partial_{\alpha} \partial_{\beta} \partial_{\gamma} F_{i}(\boldsymbol{d})=-\partial_{\alpha} \partial_{\beta} \partial_{\gamma} \partial_{i} V(\boldsymbol{d}) .
$$


The first order variational equations $\mathrm{VE}_{1}$ given by 22 have the form of a direct product. Thus we have a perfect splitting of the problem. In order to perform effectively analysis of $\mathrm{VE}_{2}$ and $\mathrm{VE}_{3}$ we have to split the problem into smaller subsystems. We can do this in the following way. We set to zero all the variables $q_{i, 1}$ except $q_{\alpha, 1}$ in the system (22)-(23). We get a system of $n$ independent subsystems of $\mathrm{VE}_{2}$ which we denote by $\mathrm{VE}_{2, \alpha}^{\gamma}$, for $1 \leq \gamma \leq n$. Such a system has the form

$$
\left.\begin{array}{l}
\ddot{q}_{\alpha, 1}=-\lambda_{\alpha} \varphi(t)^{k-2} q_{\alpha, 1}, \\
\ddot{q}_{\gamma, 2}=-\lambda_{\gamma} \varphi(t)^{k-2} q_{\gamma, 2}+\varphi(t)^{k-3} \theta_{\alpha, \alpha}^{\gamma} q_{\alpha, 1}^{2} .
\end{array}\right\}
$$

We denote by $\mathrm{PV}_{2, \alpha}^{\gamma}$ the corresponding Picard-Vessiot extension. In a similar way, for two fixed indices $\alpha \neq \beta$, we distinguish other $n$ subsystems $\mathrm{VE}_{2,(\alpha, \beta)}^{\gamma}$ of $\mathrm{VE}_{2}$. They are subsystems of $22-23$ obtained by putting $q_{i, 1}=0$ except for $i \in\{\alpha, \beta\}$. They are of the form

$$
\left.\begin{array}{l}
\ddot{q}_{\alpha, 1}=-\lambda_{\alpha} \varphi(t)^{k-2} q_{\alpha, 1}, \\
\ddot{q}_{\beta, 1}=-\lambda_{\beta} \varphi(t)^{k-2} q_{\beta, 1}, \\
\ddot{q}_{\gamma, 2}=-\lambda_{\gamma} \varphi(t)^{k-2} q_{\gamma, 2}+\varphi(t)^{k-3}\left[\theta_{\alpha, \alpha}^{\gamma} q_{\alpha, 1}^{2}+2 \theta_{\alpha, \beta}^{\gamma} q_{\alpha, 1} q_{\beta, 1}+\theta_{\beta, \beta}^{\gamma} q_{\beta, 1}^{2}\right] .
\end{array}\right\}
$$

Let us fix three indices $\alpha, \beta, \gamma \in\{1, \ldots, n\}$ such that $\alpha \neq \beta$, and consider the "extracted" system $\operatorname{EX}_{2,(\alpha, \beta)}^{\gamma}$ of the form

$$
\left.\begin{array}{l}
\ddot{q}_{\alpha, 1}=-\lambda_{\alpha} \varphi(t)^{k-2} q_{\alpha, 1}, \\
\ddot{q}_{\beta, 1}=-\lambda_{\beta} \varphi(t)^{k-2} q_{\beta, 1}, \\
\ddot{q}_{\gamma, 2}=-\lambda_{\gamma} \varphi(t)^{k-2} q_{\gamma, 2}+2 \varphi(t)^{k-3} \theta_{\alpha, \beta}^{\gamma} q_{\alpha, 1} q_{\beta, 1} .
\end{array}\right\}
$$

Although this system is not a subsystem of $\mathrm{VE}_{2,(\alpha, \beta)}^{\gamma}$, it is not difficult to prove the following fact.

Proposition 2.1. The differential Galois group $G_{2}=\mathrm{Gal}\left(\mathrm{VE}_{2}\right)$ is virtually Abelian iff groups $\operatorname{Gal}\left(\mathrm{VE}_{2, \alpha}^{\gamma}\right)$ and $\operatorname{Gal}\left(\operatorname{EX}_{2,(\alpha, \beta)}^{\gamma}\right)$ with $\alpha, \beta, \gamma \in\{1, \ldots, n\}$ and $\alpha \neq \beta$, are virtually Abelian.

In order to prove this proposition concerning $\mathrm{Gal}\left(\mathrm{VE}_{2, \alpha}^{\gamma}\right)$ one can invoke the same arguments as in Section 1.4 of [3]. Namely, the restriction to a subsystem gives a surjective morphism of the differential Galois group of the whole system and the differential Galois group of its subsystem.

To prove the statement of Proposition 2.1 concerning $\operatorname{Gal}\left(\mathrm{EX}_{2,(\alpha, \beta)}^{\gamma}\right)$ we use the following observation. We consider a linear non-homogeneous system

$$
\dot{\boldsymbol{x}}=\boldsymbol{A} \boldsymbol{x}+\boldsymbol{B}_{1}+\boldsymbol{B}_{2}, \quad \boldsymbol{x} \in K^{n},
$$

where $K$ is a differential field, and $\boldsymbol{A} \in \mathbb{M}(n, K), \boldsymbol{b}_{i} \in K^{n}$ for $i=1,2$. Let $\boldsymbol{x}_{1}, \boldsymbol{x}_{2} \in K^{n}$, satisfy

$$
\dot{\boldsymbol{x}}_{i}=\boldsymbol{A} \boldsymbol{x}_{i}+\boldsymbol{B}_{i} \quad \text { for } \quad i=1,2 .
$$

Then $\widehat{\boldsymbol{x}}=\boldsymbol{x}_{1}+\boldsymbol{x}_{2}$, is a particular solution of $(32)$.

Let $\widehat{L} / K$ and $\widehat{L}_{i} / K$ denotes the Picard-Vessiot extensions of $(32)$, and $(32)$, respectively. By $\widehat{G}$ and $G_{i}$ we denote the corresponding differential Galois groups. By the above 
observation we have the inclusion $\widehat{L} \subset L_{1} \cdot L_{2}$, where the product denotes the composition of fields. Hence, $\widehat{G}$ is an algebraic subgroup of $G_{1} \times G_{2}$. Thus if $G_{1}$ and $G_{2}$ are virtually Abelian, then $\widehat{G}$ is virtually Abelian. Moreover, if $\widehat{L}=L_{1} \cdot L_{2}$, then we have also the inverse implication.

Now, we have to find the necessary and sufficient conditions which guarantee that the groups $\operatorname{Gal}\left(\mathrm{VE}_{2, \alpha}^{\gamma}\right)$ and $\operatorname{Gal}\left(\mathrm{EX}_{2,(\alpha, \beta)}^{\gamma}\right)$ are virtually Abelian.

3. Some theoretical facts. Hereafter we assume that all differential fields considered have the same algebraically closed field of constants $C$.

Let us recall that the Picard-Vessiot ring $T(F / K)$ of a Picard-Vessiot extension $F / K$ is the set of those elements of $F$ which are holonomic over $K$, i.e, the elements $y \in F$ such that $y$ is a solution of a certain linear differential equation with coefficients in $K$.

In our considerations a fundamental role is played by the concept of second level integrals.

Definition 3.1. Let $F_{1} / K$ and $F_{2} / K$ be Picard-Vessiot extensions, and $F_{1} \subset F_{2}$. An element $\Phi \in F_{2}$ such that $\Phi^{\prime} \in T\left(F_{1} / K\right)$ is called an integral of the second level. Similarly, if moreover $\Phi^{\prime} \in K$ (i.e, $\Phi$ is a primitive integral over $K$ ), we say that $\Phi$ is an integral of the first level.

Obviously, a first level integral is an integral of the second level. Moreover, let us observe that any second level integral is holonomic over $K$, hence belongs to $T\left(F_{2} / K\right)$.

In what follows, we frequently meet the following tower of Picard-Vessiot extensions

$$
K \subset F_{1} \subset F_{2},
$$

with $F_{2} / F_{1}$ generated by second level integrals $\Phi_{1}, \ldots, \Phi_{q}$. Hence, the group $H:=$ $\operatorname{Gal}\left(F_{2} / F_{1}\right)$ is a vector group, and if the generating integrals are independent over $F_{1}$, then it is isomorphic to $\mathbb{C}^{q}$. We therefore get the following exact sequence of algebraic groups:

$$
0 \longrightarrow H=\mathbb{C}^{q} \longrightarrow \operatorname{Gal}\left(F_{2} / K\right) \longrightarrow \operatorname{Gal}\left(F_{1} / K\right) \longrightarrow 0 .
$$

Moreover, if we denote by $\widetilde{K}$ the algebraic closure of $K$ in $F_{1}$, the former coincides with the algebraic closure of $K$ in $F_{2}$.

In [4] we proved the following theorem which allows to decide whether the differential Galois group of extensions $F_{2} / K$ is virtually Abelian.

THEOREM 3.2. Let $K \subset F_{1} \subset F_{2}$ be a tower of Picard-Vessiot extensions such that $F_{2} / F_{1}$ is generated by integrals of the second level over $K$. Then $G_{2}:=\operatorname{Gal}\left(F_{2} / K\right)$ is virtually Abelian iff $G_{1}:=\operatorname{Gal}\left(F_{1} / K\right)$ is virtually Abelian and any second level integrals $\Phi \in F_{2}$ can be expanded in the form

$$
\Phi=R_{1}+J, \quad \text { with } \quad R_{1} \in T\left(F_{1} / K\right) \quad \text { and } \quad J^{\prime} \in \widetilde{K},
$$

where $\widetilde{K}$ is the algebraic closure of $K$ in $F_{1}$.

We can interpret this result in the following way. The fact that $\mathrm{Gal}^{\circ}\left(F_{2} / K\right)$ is Abelian implies that any given second level integral can be computed thanks to the first level integral and the exponential of integrals over $\widetilde{K}$. 
The following two lemmas can be deduced from the above theorem and they will be useful to find obstructions to integrability.

Lemma 3.3. Let $L / K$ and $L_{1} / K$ be Picard-Vessiot extensions with $L_{1} \subset L$, and $L_{1}=$ $K\left(I_{1}, \ldots, I_{s}\right)$, where $I_{i}^{\prime} \in K$, for $i=1, \ldots, s$. Assume that there exists a $C$-linear combination $I$ of these integrals $I_{i}$ which is transcendental over $K$. Assume further that there exist $\Phi \in L$ and $w \in K^{\star}$ such that

$$
\Phi^{\prime}=w I, \quad \text { that is, } \Phi=\int w I .
$$

Then the Galois group $\operatorname{Gal}(L / K)$ is virtually Abelian iff there exists a constant c such that

$$
c I-\int w \in K .
$$

Equivalently, $\Phi$ can be computed thanks to a closed formula of the form

$$
\Phi=P(I)+J \quad \text { with } \quad P(I):=\frac{c}{2} I^{2}+g I \in K[I], \quad \text { and } \quad J^{\prime} \in K .
$$

Lemma 3.4. Let $L / K$ and $L_{1} / K$ be Picard-Vessiot extensions with $L_{1} \subset L$, and $L_{1}=$ $K\left(E_{1}, \ldots, E_{s}\right)$, where $E_{i}^{\prime} / E_{i} \in K$, for $i=1, \ldots, s$. Assume that $L$ contains an element

$$
\Phi:=\int \sum_{i=1}^{r} w_{i} M_{i},
$$

where $w_{i} \in K^{\star}$, and

$$
M_{i}=M_{i}\left(E_{1}, \ldots, E_{s}\right) \in \mathbb{C}\left[E_{1}, E_{1}^{-1}, \ldots, E_{s}, E_{s}^{-1}\right],
$$

is a monomial, for $1 \leq i \leq r$. Moreover, $M_{1}, \ldots, M_{r}$ are not mutually proportional, i.e.,

$$
\frac{M_{i}}{M_{j}} \notin K \quad \text { for } \quad i \neq j .
$$

Then, we have:

1. Each separate integral $\Phi_{i}:=\int w_{i} M_{i} \in L$.

2. If the extension $L / L_{1}$ is generated by $\Phi$, then $L / K$ is virtually Abelian iff for each $1 \leq i \leq r$, there exists $c_{i} \in \mathbb{C}$, such that

$$
\frac{\Phi_{i}+c_{i}}{M_{i}} \in K
$$

For a proof we refer the reader to [4].

4. The second order variational equations for $k=2$. According to Proposition 2.1. in order to find necessary and sufficient conditions which guarantee that $\mathrm{Gal}\left(\mathrm{VE}_{2}\right)$ is virtually Abelian, we have to decide whether the differential Galois groups of $\mathrm{VE}_{2, \alpha}^{\gamma}$ and $\mathrm{EX}_{2,(\alpha, \beta)}^{\gamma}$ are virtually Abelian.

In order to simplify notations it is convenient to re-scale the problem. First, we notice that if the Darboux point satisfies $V^{\prime}(\boldsymbol{d})=\gamma \boldsymbol{d}$, then $\boldsymbol{d}$ is an eigenvector of $V^{\prime \prime}(\boldsymbol{d})$ with the corresponding eigenvalues $\lambda_{n}=\gamma(k-1)$. Clearly, it is convenient to set $\gamma=1$. For $k \neq 2$ we can always do this, because if $\boldsymbol{d}$ satisfies $V^{\prime}(\boldsymbol{d})=\gamma \boldsymbol{d}$, then $\widetilde{\boldsymbol{d}}:=\delta \boldsymbol{d}$, with $\delta$ chosen such that $\delta^{k-2} \gamma=1$, satisfies $V^{\prime}(\widetilde{\boldsymbol{d}})=\widetilde{\boldsymbol{d}}$. 
For $k=2$ this trick does not work. However, in this case if the Darboux point satisfies $V^{\prime}(\boldsymbol{d})=\gamma \boldsymbol{d}$ with $\gamma \neq 1$, then we replace the potential $V(\boldsymbol{q})$ by $V(\boldsymbol{q}) / \gamma$. As this change is equivalent to a re-parametrisation of the time, it does not change the integrability property of the system. That is, the system with the potential $V(\boldsymbol{q})$ is integrable iff the system with the potential $V(\boldsymbol{q}) / \gamma$ is integrable.

As we consider only one Darboux point, we can assume, without loss of generality, that $\gamma=\omega_{n}=1$.

We fix the energy of particular solution to $e=1 / 2$. Now, since $k=2$, a particular solution $\varphi(t)$ with energy $e=1 / 2$ satisfies $\dot{\varphi}^{2}+\varphi^{2}=1$, see 11. Thus, we can take, e.g., $\varphi(t)=\sin t$.

The first order variational equation

$$
\ddot{\boldsymbol{q}}_{1}:=-\varphi^{k-2} V^{\prime \prime}(\boldsymbol{d}) \boldsymbol{q}_{1}=-V^{\prime \prime}(\boldsymbol{d}) \boldsymbol{q}_{1}
$$

is a matrix second order equation over the ground differential field $K=\mathbb{C}(\varphi, \dot{\varphi})=\mathbb{C}\left(\mathrm{e}^{\mathrm{i} t}\right)$. We rewrite it as a first order equation

$$
\dot{\boldsymbol{x}}_{1}=\boldsymbol{A} \boldsymbol{x}_{1}, \quad \text { where } \quad \boldsymbol{A}:=\left[\begin{array}{cc}
\mathbf{0}_{n} & \boldsymbol{E}_{n} \\
-V^{\prime \prime}(\boldsymbol{d}) & \mathbf{0}_{n}
\end{array}\right] \in \operatorname{sp}(2 n, \mathbb{C}) .
$$

It is easy to show that the matrix $\boldsymbol{A}$ has eigenvalues $\pm \mathrm{i} \omega$, where $\omega^{2}=\lambda$, and $\lambda$ is an eigenvalue of $V^{\prime \prime}(\boldsymbol{d})$. Thus, the entries of the fundamental matrix $\boldsymbol{X}_{1}$ of equation (37) belong to a ring of the form

$$
R_{1}:=\mathbb{C}\left(\mathrm{e}^{\mathrm{i} t}\right)\left[\mathrm{e}^{ \pm \mathrm{i} \omega_{1} t} ; \ldots ; \mathrm{e}^{ \pm \mathrm{i} \omega_{n} t} ; t\right],
$$

where $\omega_{1}^{2}, \ldots, \omega_{n}^{2}$ are the eigenvalues of $V^{\prime \prime}(\boldsymbol{d})$.

With this notation and assumptions the $\mathrm{VE}_{2, \alpha}^{\gamma}$ can be rewritten in the form

$$
\left.\begin{array}{l}
\ddot{x}=-\omega_{\alpha}^{2} x \\
\ddot{z}=-\omega_{\gamma}^{2} z+\frac{\theta_{\alpha, \alpha}^{\gamma}}{\sin t} x^{2},
\end{array}\right\}
$$

where, to simplify notation, instead of blind variables $q_{\alpha, 1}$ and $q_{\gamma, 2}$, we introduce $x$ and $z$. In a similar way we rewrite the $\mathrm{EX}_{2, \alpha}^{\gamma}$ in the form

$$
\left.\begin{array}{l}
\ddot{x}=-\omega_{\alpha} x, \\
\ddot{y}=-\omega_{\beta} y, \\
\ddot{z}=-\omega_{\gamma} z+2 \frac{\theta_{\alpha, \beta}^{\gamma}}{\sin t} x y .
\end{array}\right\}
$$

The last equations in $\sqrt{\mathrm{VE}_{2, \alpha}^{\gamma}}$ and $\mathrm{EX}_{2,(\alpha, \beta)}^{\gamma}$ have the same form

$$
\ddot{z}=-\omega^{2} z+\frac{b(t)}{\sin t}
$$

with $\mathrm{e}^{\mathrm{i} \omega t}$ and $b(t)$ belonging to $R_{1}$.

Now, we consider equation (39) over the field $K=\mathbb{C}\left(\mathrm{e}^{\mathrm{i} t}\right)$. Our aim is compute the Picard-Vessiot extension $L / K$. Let $L_{1}$ be the Picard-Vessiot extension of $K$ containing $b(t), \dot{b}(t)$, and all solutions of $\ddot{z}=-\omega^{2} z$. Since $b(t)$ is holonomic over $K$, it belongs to the Picard-Vessiot ring $T\left(L_{1} / K\right)$. Moreover, according to the classical method of variation 
of constants, the extension $L / L_{1}$ is generated by the second level integrals over $K$. These integrals are described in the following lemma.

LEMMA 4.1. With the above notation the following statements hold true:

1. If $\omega=0$, then $L / L_{1}$ is generated by

$$
\int \frac{b(t)}{\sin t} \mathrm{~d} t \quad \text { and } \quad \int \frac{t b(t)}{\sin t} \mathrm{~d} t
$$

2. If $\omega \neq 0$, then $L / L_{1}$ is generated by

$$
\int \frac{\mathrm{e}^{\mathrm{i} \omega t} b(t)}{\sin t} \mathrm{~d} t \quad \text { and } \quad \int \frac{\mathrm{e}^{-\mathrm{i} \omega t} b(t)}{\sin t} \mathrm{~d} t
$$

The following two lemmas give necessary and sufficient conditions which guarantee that the groups $\operatorname{Gal}\left(\mathrm{VE}_{2, \alpha}^{\gamma}\right)$ and $\operatorname{Gal}\left(\mathrm{EX}_{2,(\alpha, \beta)}^{\gamma}\right)$ are virtually Abelian.

Lemma 4.2. The Galois group $\mathrm{Gal}\left(\mathrm{VE}_{2, \alpha}^{\gamma}\right)$ is virtually Abelian iff either $\theta_{\alpha, \alpha}^{\gamma}=0$, or $\theta_{\alpha, \alpha}^{\gamma} \neq 0$, and $\omega_{\alpha}, \omega_{\gamma} \in \mathbb{Q}^{*}$.

LEMma 4.3. The Galois group $\operatorname{Gal}\left(\mathrm{EX}_{2,(\alpha, \beta)}^{\gamma}\right)$ is virtually Abelian iff either $\theta_{\alpha, \beta}^{\gamma}=0$, or $\theta_{\alpha, \beta}^{\gamma} \neq 0$, and $\omega_{\alpha}, \omega_{\beta}, \omega_{\gamma} \in \mathbb{Q}^{\star}$.

In order to prove the above lemmas we use basic Lemma 3.3 and 3.4 The key point is to show that integrals of the form (40) and (41) are not algebraic over the field of meromorphic functions on $\mathbb{C}$.

5. Outline of proof of Theorem 1.2. In order to see how to prove this theorem, we first show what can be deduced from the assumptions just by considering the $\mathrm{VE}_{2}$.

We assumed in the theorem the existence of a Darboux point $\boldsymbol{d}$ satisfying $V^{\prime}(\boldsymbol{d})=\boldsymbol{d}$. Moreover, we assumed also that $V^{\prime \prime}(\boldsymbol{d})=\operatorname{diag}\left(\omega_{1}^{2}, \ldots, \omega_{n-1}^{2}, \omega_{n}^{2}\right)$, with $\omega_{n}=1$. The non-resonance condition 13 implies that $\omega_{i} \notin \mathbb{Q}$. Now, because $V^{\prime \prime}(\boldsymbol{d}) \boldsymbol{d}=\boldsymbol{d}$, we have $\boldsymbol{d}=[0, \ldots, 0,1]^{T}$.

Applying Lemma 4.2 to $\mathrm{VE}_{2, \alpha}^{\gamma}$, we obtain that $\theta_{2, \alpha}^{\gamma}=0$, for all $\alpha, \gamma \in\{1, \ldots, n-1\}$ except $\alpha=\gamma=n$. Recall that $\theta_{2, \alpha}^{\gamma}$ are proportional to appropriate partial derivatives of $V$ calculated at $\boldsymbol{d}$, see $(26)$. In this way we proved that

$$
\partial_{\alpha}^{2} \partial_{\gamma} V(\boldsymbol{d})=0
$$

for all $\alpha, \gamma \in\{1, \ldots, n-1\}$, except for $\alpha=\gamma=n$.

In a similar way, applying Lemma 4.2 to $\operatorname{EX}_{2,(\alpha, \beta)}^{\gamma}$, we prove that

$$
\partial_{\alpha} \partial_{\beta} \partial_{\gamma} V(\boldsymbol{d})=0
$$

for all $\alpha, \beta, \gamma \in\{1, \ldots, n-1\}$, except for $\alpha=\beta=\gamma=n$.

We showed that all third order partial derivatives of $V$ evaluated at $\boldsymbol{d}$, except for $\partial_{n}^{3} V(\boldsymbol{d})$, vanish. But the second order partial derivatives of $V$ are homogeneous functions of degree zero. Using the Euler identity, we deduce that if at least one of the indices $\alpha$, $\beta$, or $\gamma$ is equal to $n$, then $\partial_{\alpha} \partial_{\beta} \partial_{\gamma} V(\boldsymbol{d})=0$. Hence, also $\partial_{n}^{3} V(\boldsymbol{d})=0$. As a consequence, all third order partial derivatives of $V$ evaluated at $\boldsymbol{d}$ vanish, and $\mathrm{VE}_{2}$ is a copy of $\mathrm{VE}_{1}$, see (23). In this way, the non-homogeneous term in $\mathrm{VE}_{3}$, see 20), depends only on solutions of $\mathrm{VE}_{1}$. 
Thus, it is clear that we can try to prove the theorem using induction. In order to describe the general step of this induction we represent $V$ as a power series

$$
V(\boldsymbol{q})=\sum_{|\boldsymbol{\alpha}| \geq 0}^{\infty} \frac{1}{\boldsymbol{\alpha} !} \partial^{\boldsymbol{\alpha}} V(\boldsymbol{d}) \cdot \widetilde{\boldsymbol{q}}^{\boldsymbol{\alpha}}
$$

where $\widetilde{\boldsymbol{q}}=\boldsymbol{q}-\boldsymbol{d}=\left(q_{1}, \ldots, q_{n-1}, q_{n}-1\right)$. In the above we used the standard multi-index notation. That is

$$
\boldsymbol{\alpha}=\left(\alpha_{1}, \ldots, \alpha_{n}\right) \in \mathbb{N}^{n}, \quad|\boldsymbol{\alpha}|:=\sum_{i=1}^{n} \alpha_{i}, \quad \boldsymbol{\alpha} !=\alpha_{1} ! \cdots \alpha_{n} !, \quad \boldsymbol{q}^{\boldsymbol{\alpha}}:=q_{1}^{\alpha_{1}} \cdots q_{n}^{\alpha_{n}},
$$

and

$$
\partial^{\boldsymbol{\alpha}}:=\partial_{1}^{\alpha_{1}} \cdots \partial_{n}^{\alpha_{n}}, \quad \text { where } \quad \partial_{i}^{\alpha_{i}}:=\frac{\partial^{\alpha_{i}}}{\partial q_{i}^{\alpha_{i}}} .
$$

Taking into account that $V^{\prime}(\boldsymbol{d})=\boldsymbol{d}$, we have also by the Euler identity $V(\boldsymbol{d})=1 / 2$, and the above expansion can be written in the form

$$
V(\boldsymbol{q})=\frac{1}{2}\left(\omega_{1}^{2} q_{1}^{2}+\cdots+\omega_{n-1}^{2} q_{n-1}^{2}+q_{n}^{2}\right)+\sum_{|\boldsymbol{\alpha}| \geq 3} \frac{1}{\boldsymbol{\alpha} !} \partial^{\boldsymbol{\alpha}} V(\boldsymbol{d}) \cdot \widetilde{\boldsymbol{q}}^{\boldsymbol{\alpha}} .
$$

Thus, we have to prove that

$$
\partial^{\boldsymbol{\alpha}} V(\boldsymbol{d})=0, \quad \text { for all } \boldsymbol{\alpha} \text { with }|\boldsymbol{\alpha}|=m \geq 3 .
$$

The vanishing of all these coefficients in the Taylor expansion given by (45), is going to be made by successive use of the $\mathrm{VE}_{p}$ and justify the title of our paper.

Let us assume that it is true for $3 \leq m \leq p$. Then $\mathrm{VE}_{p}$ has the following simple form:

$$
\left.\begin{array}{l}
\ddot{x}_{i}=-\omega_{i}^{2} x_{i}, \quad 1 \leq i \leq n, \\
\left.\ddot{y}_{j}=-\omega_{j}^{2} y_{j}+\sum_{|\boldsymbol{\alpha}|=p} \frac{\xi_{\boldsymbol{\alpha}}^{j}}{\sin ^{p-1}(t)} \boldsymbol{x}^{\boldsymbol{\alpha}}, \quad 1 \leq j \leq n, \quad\right\}
\end{array}\right\}
$$

where $\boldsymbol{x}=\left(x_{1}, \ldots, x_{n}\right)$, and

$$
\xi_{\boldsymbol{\alpha}}^{j}:=\frac{1}{\boldsymbol{\alpha} !} \partial^{\boldsymbol{\alpha}} F_{j}(\boldsymbol{d})
$$

To prove the inductive step we have to show that $\xi_{\boldsymbol{\alpha}}^{j}=0$ for $j=1, \ldots n$, and all multiindices such that $|\boldsymbol{\alpha}|=p$. To this end we show first that we can apply Lemma 3.4 in this case.

Let $K=\mathbb{C}\left(\mathrm{e}^{\mathrm{i} t}\right)$ be our ground field, and $L:=\mathrm{PV}_{p}$ be the Picard-Vessiot extension of $\mathrm{VE}_{p}$ over $K$. The field $L$ contains

$$
L_{1}:=\mathrm{PV}_{1}=K\left(\mathrm{e}^{\mathrm{i} \omega_{1} t}, \ldots, \mathrm{e}^{\mathrm{i} \omega_{n-1} t}\right),
$$

According to Lemma 4.1 the field $L$ contains a second level integral of the form

$$
\Phi_{j}=\int \sum_{|\boldsymbol{\alpha}|=p} \frac{\xi_{\boldsymbol{\alpha}}^{j} \mathrm{e}^{\mathrm{i} \omega_{j} t}}{\sin ^{p-1}(t)} \boldsymbol{x}^{\boldsymbol{\alpha}}
$$

In particular if we choose

$$
\boldsymbol{x}=\left(x_{1}, \ldots, x_{n}\right)=\left(\mathrm{e}^{\mathrm{i} \omega_{1} t}, \ldots, \mathrm{e}^{\mathrm{i} \omega_{n} t}\right), \text { with } \omega_{n}=1,
$$


then

$$
\boldsymbol{x}^{\boldsymbol{\alpha}}=\mathrm{e}^{\mathrm{i}\left(\alpha_{1} \omega_{1}+\cdots+\alpha_{n} \omega_{n}\right) t}=\mathrm{e}^{\mathrm{i}(\boldsymbol{\alpha} \cdot \boldsymbol{\omega}) t},
$$

and $L$ contains an integral of the form

$$
\Phi_{j}=\sum_{|\boldsymbol{\alpha}|=p} \xi_{\boldsymbol{\alpha}}^{j} \int \frac{1}{\sin ^{p-1}(t)} \mathrm{e}^{\mathrm{i}\left(\boldsymbol{\alpha} \cdot \boldsymbol{\omega}+\omega_{j}\right) t}=\sum_{|\boldsymbol{\alpha}|=p} \xi_{\boldsymbol{\alpha}}^{j} T_{p-1, \boldsymbol{\alpha} \cdot \boldsymbol{\omega}+\omega_{j}},
$$

where

$$
T_{r, \omega}:=\int \frac{\mathrm{e}^{\mathrm{i} \omega t}}{\sin ^{r}(t)},
$$

for $r \in \mathbb{Z}$ and $\omega \in \mathbb{C}$. In order to apply Lemma 3.4 we consider the monomials

$$
M_{\boldsymbol{\alpha}}:=\mathrm{e}^{\mathrm{i}\left(\boldsymbol{\alpha} \cdot \boldsymbol{\omega}+\omega_{j}\right) t},
$$

with fixed $j$. As $\omega_{1}, \ldots, \omega_{n}$ are $\mathbb{Z}$ independent, one can easily prove that they are not mutually proportional. So, we can apply Lemma 3.4 Now, according to the first point of this lemma we have that each term in the sum 48 is an element of $L$, i.e.,

$$
\xi_{\boldsymbol{\alpha}}^{j} T_{p-1, \boldsymbol{\alpha} \cdot \boldsymbol{\omega}+\omega_{j}} \in L .
$$

Moreover, according to the second point of Lemma 3.4. we know that if $\operatorname{Gal}(L / K)$ is virtually Abelian, then there exists a constant $c$ such that

$$
\frac{\xi_{\boldsymbol{\alpha}}^{j} T_{p-1, \boldsymbol{\alpha} \cdot \boldsymbol{\omega}+\omega_{j}}+c}{\mathrm{e}^{\mathrm{i}\left(\boldsymbol{\alpha} \cdot \boldsymbol{\omega}+\omega_{j}\right) t}} \in K .
$$

It follows that $\xi_{\boldsymbol{\alpha}}^{j} T_{p-1, \boldsymbol{\alpha} \cdot \boldsymbol{\omega}+\omega_{j}}$ is meromorphic on $\mathbb{C}$. But one can show that $T_{p-1, \boldsymbol{\alpha} \cdot \boldsymbol{\omega}+\omega_{j}}$ is not meromorphic, and hence $\xi_{\boldsymbol{\alpha}}^{j}=0$. This is the case for $j \in\{1, \ldots, n\}$ and multi-indices $\boldsymbol{\alpha}$ such that $|\boldsymbol{\alpha}|=p$ satisfying the condition $\left(\boldsymbol{\alpha} \cdot \boldsymbol{\omega}+\omega_{j}\right) \in \mathbb{Z}$. Thanks to the non-resonance assumption this condition is fulfilled iff $j=n$ and $\boldsymbol{\alpha}=\boldsymbol{\alpha}_{0}:=(0, \ldots, 0, p)$. However, using the Euler identity one can easily show that also $\xi_{\boldsymbol{\alpha}_{0}}^{j}=0$, and this finishes the proof of the inductive step.

Acknowledgements. We are thankful to Thierry Combot for sending his unpublished papers concerning applications of higher order variational equations to a study of the integrability of homogeneous potentials.

The second author is thankful to European Science Foundation (ESF) for grant for participation in the conference "Algebraic Methods in Dynamical Systems" in Będlewo.

\section{References}

[1] A. D. Bruno, The Restricted 3-body Problem: Plane Periodic Orbits, de Gruyter Expositions in Mathematics 17, Walter de Gruyter, Berlin, 1994.

[2] G. Casale, Morales-Ramis theorems via Malgrange pseudogroup, Annales de l'Institut Fourier 59 (2009), 2593-2610.

[3] G. Duval and A. J. Maciejewski, Jordan obstruction to the integrability of Hamiltonian systems with homogeneous potentials, Annales de l'Institut Fourier 59 (2009), 2839-2890.

[4] G. Duval and A. J. Maciejewski, Higher order variational equations for Hamiltonian systems with homogeneous potentials I, 2011, in preparation. 
[5] J. J. Morales-Ruiz and J. P. Ramis, Galoisian obstructions to integrability of Hamiltonian systems. I, Methods Appl. Anal. 8 (2001), 33-95.

[6] J. J. Morales-Ruiz and J. P. Ramis, Galoisian obstructions to integrability of Hamiltonian systems. II, Methods Appl. Anal. 8 (2001), 97-111.

[7] J. J. Morales-Ruiz and J. P. Ramis, Integrability of dynamical systems through differential Galois theory: a practical guide, in: Differential Algebra, Complex Analysis and Orthogonal Polynomials, Contemp. Math. 509, Amer. Math. Soc., Providence, RI, 2010, 143-220.

[8] J. J. Morales-Ruiz, J. P. Ramis and C. Simó, Integrability of Hamiltonian systems and differential Galois groups of higher variational equations, Ann. Sci. Éc. Norm. Supér. 40 (2006), 845-884. 
